\title{
Body movement implied by static images modulates eye movements and subjective time estimation
}

\author{
Francisco Carlos Nather ${ }^{1}$, José Lino Oliveira Bueno ${ }^{1}$, and Emmanuel Bigand ${ }^{2}$ \\ 1. Universidade de São Paulo, São Paulo, SP, Brazil \\ 2. University of Bourgogne, Dijon, Burgundy, France
}

\begin{abstract}
The present study investigated the influence of body movement implied by artwork on time estimation and its relationship with eye movement. In Experiment 1, the participants were presented with static photographic images of Edgar Degas sculptures that implied different movements. The participants were asked to estimate their exposure times. Overestimation was found for the dancer who represented the greater movement. Eye movements were more directed to legs and arms in the ballerina that implied more movement, indicating more dynamic eye movements for this dancer, which was also overestimated. Experiment 2 further investigated whether this effect was specific to body representation or whether it was linked to the perceptual feature of the images. The participants were presented with drawings that mimicked global shapes (lines) of the Degas sculptures. Differences in time estimation were not obtained under this experimental condition. The results suggest an embodied perception of body movement that modulates eye movements and time estimation. Keywords: time perception, implied movement, eye-tracking, static image, artwork.
\end{abstract}

Received 14 June 2013; received in revised form 21 October 2013; accepted 23 October 2013. Available online 23 December 2013.

\section{Introduction}

Human figures in static images have revealed different psychological processes related to the perception of time, such as the meaning of the images. Important aspects of time estimation have been related to the emotional content of the pictures (Angrilli, Cherubini, Pavese, \& Manfredini, 1997; Droit-Volet, \& Gil, 2009), pleasure and interest in an aesthetic episode (Cupchick; 1976; Cupchick, \& Gebotys,1988), action in pictures of naturalistic scenes (Moscatelli, Polito, \& Lacquaniti, 2011; Pavan, Cuturi, Maniglia, Casco, \& Campana, 2011), implied body movements in schematic drawings (Orgs, Bestmann, Schuur, \& Haggard, 2011; Tse, Intriligator, Rives, \& Cavanagh, 2004; Yamamoto, \& Miura, 2012), and artwork that represents motion as objects, paintings, and sculptures (e.g., Nather, \& Bueno, 2006, 2012a; Nather, Bueno, Bigand, \& Droit-Volet, 2011). These

F. C. Nather, J. L. O. Bueno, University of São Paulo, Department of Psychology, Brazil. E. Bigand, University of Bourgogne, LEAD CNRS, France. Correspondence regarding this article should be directed to: Francisco Carlos Nather and José Lino Oliveira Bueno, Departamento de Psicologia, FFCLRP - Universidade de São Paulo. Avenida Bandeirantes 3900, Ribeirão Preto, SP 14090-901 Brazil. Phone: +55 1636023697. Fax: +55 1636024835. E-mail: f.nather@ hotmail.com/jldobuen@fclrp.usp.br studies have shown that movement in static images, such as artwork, distorts the perception of time.

Time perception can constitute a relevant component of the comprehension of artistic appreciation (Cupchick, \& Gebotys, 1998). From this perspective, the use of static artwork that implies motion has shown that the observation of human body postures modulates time perception (Nather, \& Bueno, 2011). Images of bodies that imply more movement were estimated longer than those that imply less movement, although they were presented for the same duration. These time distortions were discussed in terms of embodiment mechanisms. When people observe artwork that implies motion, specific brain areas, such as those with mirror neurons, are activated and reconstruct the actions by simply observing the static pattern of a past action (Gallese, 2005; Freedberg, \& Gallese, 2007). The internal clock (Gibbon, Church, \& Meck, 1984) may be suggested to run faster with the embodied movement associated with different two-dimensional plane figurative implied movement perceived by another person (Nather et al., 2011). Thus, time distortions in the perceiver may be an index of empathic processes that enable the individual to understand the actions of others as a function of the timing related to the observed movements.

Nather, \& Bueno (2012a) discussed in detail why the observation of artwork, such as Edgar Degas' ballet dancers, causes different time distortions. This impressionist artist constructed his ballerinas 
by considering both movement representation and its implicit timing associated with the represented movements. According to the authors, Degas' interest was not only focused on considering his ballerinas as simple objects maintained in physical space but also focused on three-dimensional art pieces observed from various perspectives. Therefore, his ballerinas reveal biomechanical characteristics that are "paralyzed" in time once they contain implicit temporal movement suggestions that cannot be endlessly maintained.

Different human body movements demand more or less time to happen. Visual information plays a crucial role in understanding the three-dimensional space to determine the correct movement to perform an action. For example, patterns of eye movements can predict future observed actions. Because of this, the coordination between the gaze of the actions of others is similar to when the observer performs the same action himself (Flanagan, \& Johansson, 2003) because the temporal prevision of a future movement of the members is facilitated by eye movements (Gueugneu, Crognier, \& Papaxanthis, 2008). Additionally, eye movements play a functional role in the imagery of human actions. They can transform spatial information in action plans that coordinate the body members' movements (De'Sperati, \& Santandrea, 2005). The same neural mechanisms related to the eye movements of three-dimensional body movement participate in the planning of both imagined and performed body actions. However, this information is based on experiments that demanded the observer's attention to actual three-dimensional actions. The point of interest of this study was to understand the eye movements associated with the observation of two-dimensional artwork that implied different body movements.

Eye movements have been addressed with regard to different aspects of visual perception that involve attentional factors and psychological processes that relate these movements to scene perception (Duchowski, 2007; Rayner, 1998), the visual perception of artwork (Mannan, Ruddock, \& Wooding, 1997; Wooding, 2002), and expertise in visual arts (Nodine, Locher, \& Krupinskiet al, 1993; Vogt, \& Magnusse, 2007; Zangemeister, Sherman, \& Stark, 1995). Eye movement patterns in complex images likely reflect a collection of varying time range processes, from early sensory recognition to memory processing (Wang, Freeman, Merriam, Hasson, \& Heeger, 2012). Different cognitive systems interact with different parts of the image to determine where the eyes fixate and how long they remain fixated in a specific location (Henderson, 1993, 2003). Therefore, important conclusions related to the temporal aspects of image perception can be obtained by eye movement analyses (Rayner, 1998).

Different strategies are used by participants during the time estimation of artwork of a ballerina (Nather, 2006; Nather, \& Bueno, 2006). The majority of subjects reported "to repeat the silhouette of ballerinas visualized" and "to repeat the different body parts of ballerinas revisualizing them." Therefore, associating these time distortions with eye movement repetitions is possible, in which the time spent fixating the eyes on a specific image area is highly correlated with the time spent fixated on the same areas during their presentation (Brandt, \& Stark, 1997; Laeng, \& Teodorescu, 2002). From this perspective, information about where and how long people look at the different body parts of Degas' ballerinas will contribute to a better understanding of the processes of visual motion perception and subjective time.

Expressive qualities of movement are connected to what we know about their meaning (Arnheim, 2004). For example, the figurative image of a dancer who poses with her arms up gives the observer dynamic properties because it is perceived as a deviation from the normal standing posture. According to Arnheim, more than reference points for the eyes, the parts of the body both direct the eye and can show what the body is doing, suggesting that some properties and body functions constitute an inseparable part of its visible character. From this perspective, the use of abstract images that represent these ballerinas' bodies, including schematic body forms as black-line forms, is appropriate to discern the effects and differentiate the sensorial and figurative image components when studying subjective time using eye movement records (see Orgs et al., 2011; Orgs, Kirsch, \& Haggard, 2013).

The present study verified the relationship between eye movements and subjective time estimations of subjects exposed to different implied movement images for different time durations. In Experiment 1, the participants observed pictures of Degas' ballerina sculptures that implied movements for 18 or $36 \mathrm{~s}$. The use of different time records was justified because the patterns of similarities between eye fixation can be different with different durations of exposure, varying by a few seconds, and because time perception can be affected by the duration of exposure (Mannan et al., 1997; Nather, \& Bueno, 2012b). Experiment 2 used these same images of human bodies that were reduced to black lines to further investigate whether this effect is specific to body posture representation or whether it is linked to the perceptual features of the images.

\section{Experiment 1}

\section{Methods}

\section{Participants}

Forty-six undergraduate psychology students (six men) who were not trained in Visual Arts or Classical Ballet at the University of Bourgogne (France), aged 18 to 23 years, participated in the experiment. The participants were divided into two groups that were presented the stimuli for $18 \mathrm{~s}$ (G18 group, $n=14$ ) and $36 \mathrm{~s}(\mathrm{G} 36$ group, $n=32$ ). They reported having normal or corrected-to-normal vision and gained course credit for their participation. 


\section{Materials}

The experiment was conducted during the daytime in the Laboratoire d'Etude de l'Apprentissage et du Développement (Faculty of Psychology). The room remained with the lights off during the experiment.

Four photographs of dancer sculptures by Edgar Degas presented in geometric progression according to the Body Movement Ranking Scale (Nather, \& Bueno, 2008) were used as stimuli: "Ballerina resting, hands on hips and legs left forward" (1.5 points), "Ballerina resting with hands on hips and right leg forward" (3.0 points), "Third time of great arabesque" (6.0 points), and "First time of the great arabesque" (training stimulus; 4.0 points; Figure 1A). The final size of the images on the Tobii Studio screen was approximately $30 \times 40 \mathrm{~cm}$, with $1024 \times 768$ dpi resolution. The ballerina images occupied the central position of the computer screen, and the rest of the screen was filled with white.

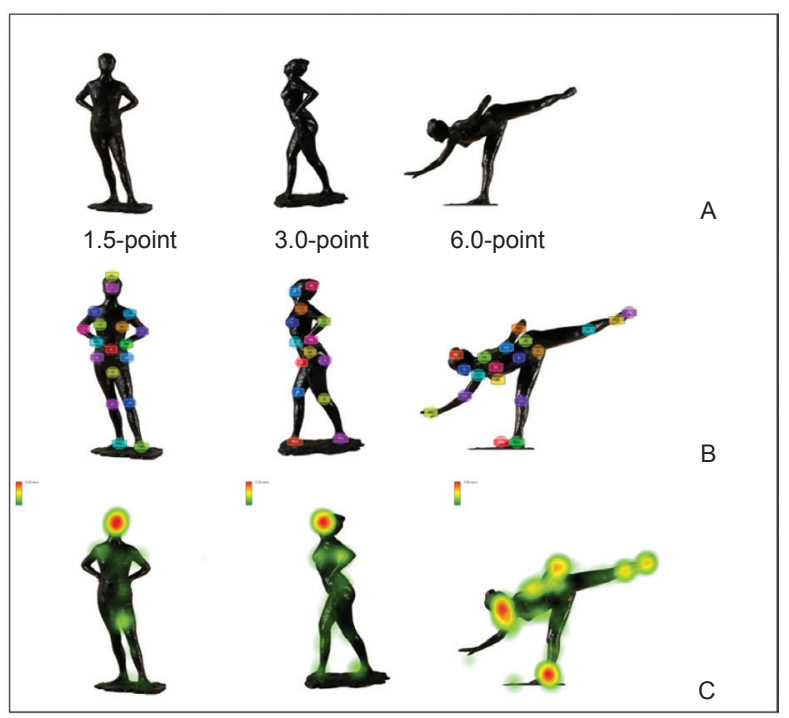

Figure 1. (A) The 1.5-, 3.0-, and 6.0-point stimuli. Notice that the 1.5- and 3.0-point stimuli are the same position but viewed from different angles. (B) AOIs used in the experiment. (C) Illustrative images of the total time of observation of the body parts for one participant in the G36 group revealed by Tobii Studio. Colors (green to red) indicate a range: the red means more time observing a specific body part, and the green means the opposite. (C) Edgar Degas, Paris, France, 1834-1917, MASP Collection, Museum of Art of São Paulo Assis Chateaubriand. Pictures by João L. Musa.

The eye movements toward the body images were recorded using Tobii Studio 1.1. The eye tracking parameters were the following: (a) fixation time (FT; the total duration of observation of a specific body part), (b) observation count (OC; how many times one area of the body was observed by the subjects), and (c) time to first fixation (TFF; the time interval elapsed since the onset of the stimulus until a given area of interest received fixation for the first time during the total time exposure of the images). Therefore, marking the different parts of the body sculptures according to eyetracking methodology was necessary (areas of interest [AOI]). These AOIs were defined according to Civilian American and European Surface Anthropometry
Resource criteria (Robinette et al., 2002). Therefore, the main joints of the body (scapular and pelvic girdle, elbows, wrists, knees, and ankles), parts of the thorax and abdomen (breasts, abdomen/umbilicus, and pelvic region), face, and head were marked as circular AOIs (Figure 1B).

The time estimations were recorded using the Wave Surfer program installed on an HP Pavilion notebook. The prospective paradigm with the reproduction method of subjective time was used for time estimations (Block, 1990).

\section{Procedure}

The participants individually entered the experimental room and were asked to sit in a chair. They were then positioned facing the central region of the Tobii Studio screen at a fixed distance of $40 \mathrm{~cm}$.

The tasks were verbally explained to the participants. They were instructed to observe each image. After each observation, they were asked to estimate its duration of presentation on the white computer screen. Each participant observed the three stimuli that were presented in random experimental sequences. Each stimulus was presented for 18 or 36 s (G18 and G36 groups, respectively). The training stimulus (4.0 points) was used first to familiarize the participants with the experimental task.

After the time estimations, a questionnaire was used to obtain personal information and the strategies used by the participants to make their time estimations.

\section{Analyses}

A one-way repeated-measures analysis of variance (ANOVA) and Student-Newman-Keuls post hoc comparisons were used to compare the time estimation data. Student's $t$-test was used to compare the mean values of the time ratios of the stimuli with the actual time of exposure (18 s for the G18 group and $36 \mathrm{~s}$ for the G36 group).

A two-way repeated-measures ANOVA and StudentNewman-Keuls post hoc comparisons were used to compare the FT and OC data (eye-tracker parameters). For these analyses, the AOI data were grouped according to the main body parts: head, torso, and members (i.e., arms and legs). The $\chi^{2}$ test was used for the analysis of the TFF parameter using all of the AOIs.

\section{Results}

\section{Time estimation}

The ANOVA did not reveal a significant difference in time estimation among the stimuli in the G18 group. The $t$-test results showed that the temporal estimation of the 1.5-, 3.0-, and 6.0-point images were not different from the actual duration of $18 \mathrm{~s}$.

In contrast, a significant effect of time estimation was observed in the G36 group $\left(F_{2,62}=3.24, p<.05\right)$. The mean values of $35.66 \mathrm{~s}$ (1.5-point) and $34.44 \mathrm{~s}$ (3.0-point) were different from $39.87 \mathrm{~s}$ (6.0-point). The 1.5- and 
3.0-point stimuli were estimated to be shorter than the 6.0 -point stimulus. The $t$-test results showed that the 6.0-point stimulus (more movement) was overestimated $\left(t_{50}=-2.56, p=.05\right)$, and the 1.5- and 3.0-point stimuli (less movement) were estimated accurately in relation to the actual duration of $36 \mathrm{~s}$.

\section{Eye movements}

Fixation time parameter (G18 group). The ANOVA of FT in the G18 group, considering the sculptures (1.5-, 3.0-, and 6.0-point stimuli) and different body parts of each sculpture (head, torso, arms, and legs), revealed a significant effect of body part $\left(F_{3,78}=8.66, p<.001\right)$ and a sculpture $\times$ body part interaction $\left(F_{6,78}=11.50\right.$, $p<.001)$.

The post hoc analysis of body parts showed that the head was more observed in the 1.5-point stimulus, and the legs were more observed in the 6.0-point stimulus $(p<.001)$. The arms were also more observed than the head and torso in the 3.0- and 6.0-point stimuli. The parts of the body did not show differences in the 3.0-point stimulus.

The post hoc analysis of the interaction between body parts and the different sculptures showed that the head was more observed in the 1.5-point stimulus than in the 3.0- and 6.0-point stimuli, but the legs were more observed in the 6.0-point stimulus than in the 1.5- and 3.0 -point stimuli $(p<.001)$. The visual observation of the torso, arms, and legs was similar in the 1.5- and 3.0-point stimuli. The arms were more observed in the 6.0 -point stimulus than in the 1.5 -point stimulus (all $p<.01)$.

Observation count parameter (G18 group). The ANOVA of the OC, considering the sculptures (1.5-, 3.0-, and 6.0-point stimuli) and different body parts of each sculpture (head, torso, arms, and legs), revealed significant effects of sculpture $\left(F_{2,78}=15.94, p<.001\right)$ and body part $\left(F_{3,78}=15.56, p<.001\right)$ and a sculpture $\times$ body part interaction $\left(F_{6,156}=12.25, p<.001\right)$. The post hoc analyses of body parts and the body part $\times$ sculpture interaction were similar to the results described for the FT parameter. In the graphical representation, the data similarity between the FT and OC parameters is evident (Figure 2).

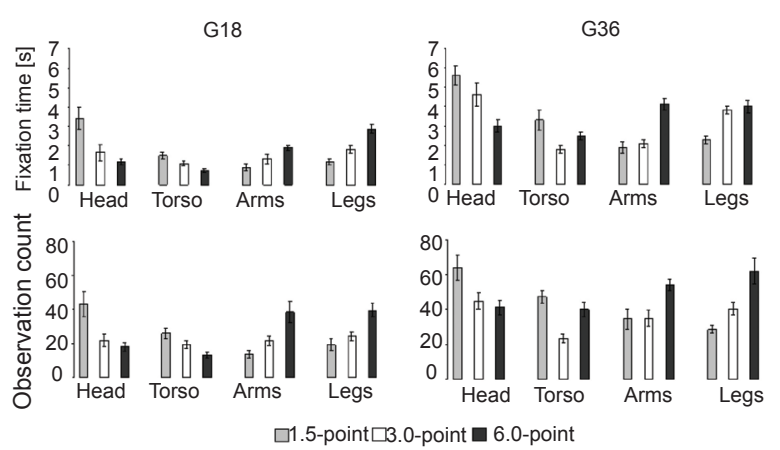

Figure 2. Mean fixation time and observation count for the head, torso, arms, and legs in the G18 and G36 groups (18 and $36 \mathrm{~s}$ exposure, respectively).
Fixation time parameter (G36 group).The data analyses for the G36 group showed similarities to the G18group (Figure 2). However, in the G36 group, eye fixation tended to be more specifically oriented to the different body parts, depending on the stimulus' implied movement.

The ANOVA of FT, considering the sculptures (1.5-, 3.0-, and 6.0-point stimuli) and different body parts of each sculpture (head, torso, arms, and legs), revealed a significant effect of body part $\left(F_{3,156}=11.30, p<.001\right)$ and a sculpture $\times$ body part interaction $\left(F_{6,156}=14.03\right.$, $p<.001)$.

The post hoc analysis showed the body parts of the three sculptures (1.5-, 3.0-, and 6.0-point stimuli) were differently observed (all $p<.05$ ). In the 1.5-point stimulus, the head was more observed than the other body parts, with the exception of the torso (all $p<.01$ ), and the arms were more observed than the torso and legs. The data analysis did not reveal different observations of the head and legs in the 3.0-point stimulus, but they were more observed than the arms and torso (all $p<.001$ ). In the 6.0-point stimulus, significant differences in head, arm, and leg observations were not found, but the arms were more observed than the torso, and they was less observed than the legs $(p<.01)$.

The post hoc analysis of the body part $\times$ sculpture interaction revealed the head was more observed in the 1.5- and 3.0-point stimuli than in the 6.0-point stimulus $(p<.001)$. Conversely, the arms and legs were more observed in the 6.0-point stimulus than in the 1.5 -point stimulus ( $p<.05$ and $p<.01$, respectively). The observation of the arms was not different between the 1.5- and 6.0-point stimuli, and the observation of the legs was not different between the 3.0- and 6.0-point stimuli. However, the legs were less observed in the 1.5 -point stimulus than in the 3.0 - and 6.0-point stimuli $(p<.001)$.

Observation count parameter (G36 group). The ANOVA of OC, considering the sculptures (1.5-, 3.0-, and 6.0-point stimuli) and different body parts of each sculpture (head, torso, arms, and legs), revealed significant effect of sculpture $\left(F_{2,155}=12.05, p<.001\right)$ and body part $\left(F_{3,155}=2.56, p<.05\right)$ and a sculpture $\times$ body part interaction $\left(F_{6,155}=9.36, p<.001\right)$.

In the G36 group, the post hoc analyses of body parts and the body part $\times$ sculpture interaction were very similar to those described for the FT parameter (Figure 2).

Time to first fixation parameter. The TFF data in the G18 and G36 groups suggested that the 1.5-and 3.0-point stimuli were more slowly observed than the 6.0-point stimulus (Figure 3). However, the statistical analyses that compared the time order of AOI observation did not reveal significant differences between stimuli in the G18 group.

In the G36 group, significant differences were found between the 1.5-point $\left(\chi_{17}^{2}=27.149, p<.05\right)$ and 3.0 -point $\left(\chi_{12}^{2}=27.149, p<.01\right)$ stimuli and the 6.0-point 
stimulus. In the G36 group, the participants spent more time observing the sculptures' body parts in the 1.5- and 3.0-point stimuli (less movement) than in the 6.0-point stimulus (more movement).
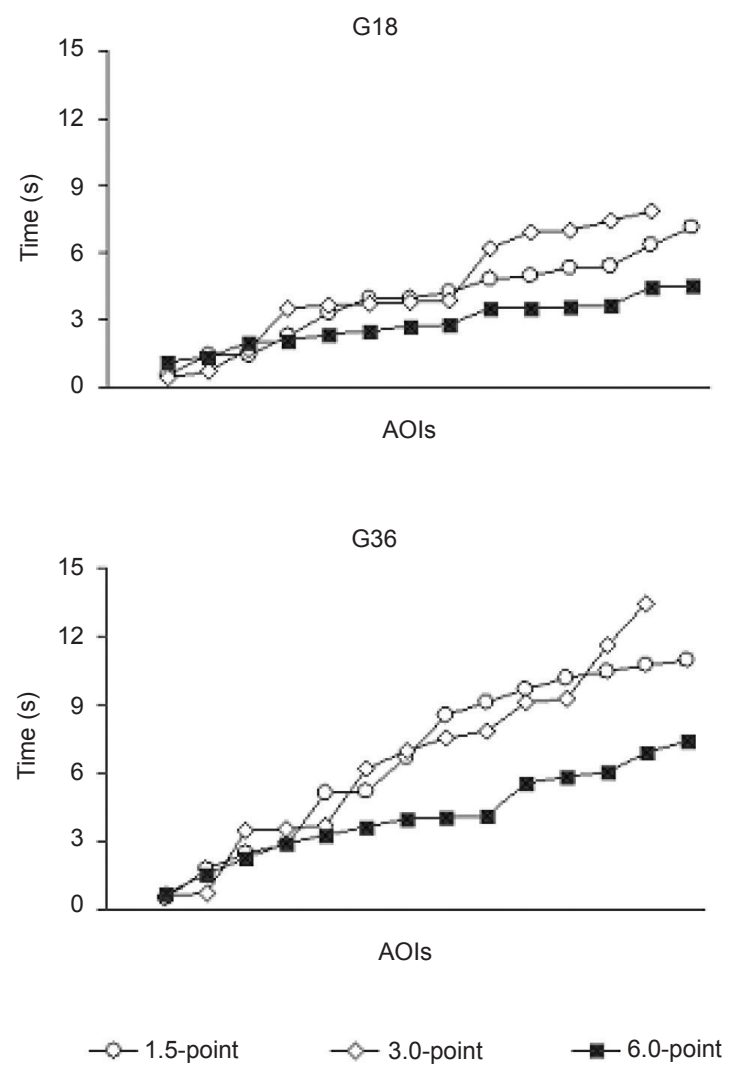

Figure 3. Mean time to first fixation for 1.5-, 3.0-, and 6.0-point stimuli in the G18 and G36 groups (18 and $36 \mathrm{~s}$ exposure, respectively).

\section{Discussion}

Time estimations observed in the G18 and G36 groups replicated the data of previous studies. Pictures of two artistic objects, a sitting doll (2.0 points of movement) and a doll that represented a dancing ballerina (5.0 points), did not affect time perception when they were exposed for $18 \mathrm{~s}$. However, exposure to these same dolls for $36 \mathrm{~s}$ showed similar time distortions as when they were observed for 36 s (Nather, 2006; Nather, \& Bueno, 2006). Similarly, the time estimation data for $36 \mathrm{~s}$ exposure were consistent with a previous study that used these same images of Degas' ballerinas (Nather, \& Bueno, 2011). The 1.5- and 3.0-point stimuli (unmoving ballerinas) were estimated shorter than the 6.0-point stimulus (dancing ballerina).

Recently, Nather, \& Bueno (2012b) suggested that the duration of stimulus exposure is a relevant parameter for subjective time studies because it is inherent in estimating time processing. In the present study, eye movements added new information about timing that were related to implicit processing using different durations of exposure of 18 and 36 s (G18 and G36 groups). The TFF data revealed that the time sequence of body part observation was not different between the three stimuli with $18 \mathrm{~s}$ exposure (G18 group), but the body parts were more slowly observed in the 1.5- and 3.0 -point stimuli in relation to the 6.0-point stimulus with 36 s exposure (G36 group; Figure 3). The patterns of eye movements appeared to be more dynamic when observing the stimulus that implied more movement for $36 \mathrm{~s}$. Therefore, the implied movements modulate eyes movements based on 1.5-, 3.0-, and 6.0-point movement intensities. Moreover, the patterns of eye movements revealed that the differences in time estimation between the G18 and G36 groups may be attributable to the duration of stimulus exposure. The distinct time distortions observed in the G36 group among the stimuli, implying smaller and greater movements, suggest that eye movements occurred as a function of the implied body movements of the ballerinas' postures.

Other eye parameters support this interpretation. The FT and OC data showed similarities in both the G18 and G36 groups. However, the data for the G36 group showed that eye fixation tended to be more specifically oriented to the different body parts, depending on the implied movement (Figure 1C). In short, in the G36 group, the head was more observed in the 1.5- and 3.0-point stimuli (less movement) than in the 6.0-point stimulus (more movement), but the arms and the legs were more observed in the 6.0-point stimulus than in the 1.5-point stimulus. Interestingly, arm observation was not different between the 1.5- and 3.0-point stimuli, and leg observation was not different between the 3.0- and 6.0-point stimuli. Therefore, the body part observation patterns in the 3.0-point stimulus were intermediary between the 1.5- and 6.0-point stimuli.

From this perspective, eye modulation was oriented by the implied body motion of the ballerinas. The legs were more observed in the 3.0- and 6.0-point stimuli, likely because the legs indicate the displacement of the body in three-dimensional space when people are standing. Importantly, the 1.5- and 3.0-point stimuli were the same body position photographed at different angles, but one of them was punctuated with 3.0 points because it can be interpreted as a ballerina walking (Figure 1). Differences in movement perception related to body oscillations were observed when impressionist paintings of Monet were rotated $180^{\circ}$ in relation to their original positions (Kapoula, \& Gaertner, 2013). Moreover, the patterns of eye movements can predict future observed actions because the temporal prevision of the body members' actions is facilitated by eye observation (Flanagan, \& Johansson, 2003; Gueugneu et al., 2008).

The main focus of Experiment 2 in the present study was to verify whether eye movements are related to the composition of image quality or whether it is related to the differential movement induction represented by body positions (Arnheim, 2004). This experiment examined whether the same body positions of Degas' ballerinas reduced to black lines affect time estimations and eye movements. In this experiment, the figurative 
content of the stimuli became more abstract because of their reduction in terms of their implicit information and comprehension (abstract forms).

\section{Experiment 2}

\section{Methods}

Experiment 2 adopted the same experimental design as Experiment 1, with the exception that only the 1.5- and 6.0-point stimuli reduced to lines were used (Figure 4). Fifty-seven psychology students (nine men) who were not trained in Visual Arts or Classical Ballet at the University of Bourgogne (France), aged between 18 and 23 years, participated in the experiment. The participants were divided into two groups who were presented with the stimuli in the original position (OP group, $n=28$ ) and inverted position (IP group, $n=29$ ). They reported having normal or corrected-to-normal vision and gained course credit for their participation.

The sculpture images were reduced to lines using Adobe Photoshop 7.0. To reduce the ballerinas' bodies to linear forms, the main joints of the bodies were marked and linked (girdle, arms, legs, wrists, etc.). To accomplish this, the same points of the bodies according to Civilian American and European Surface Anthropometry Resource criteria in Experiment 1 were used in Experiment 2 (Robinette et al., 2002). Therefore, the stimuli were constructed by adding the joints of the ballerinas' bodies by connecting them vertically using straight lines (Figure 4).

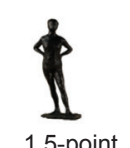

$$
\stackrel{i}{i}
$$
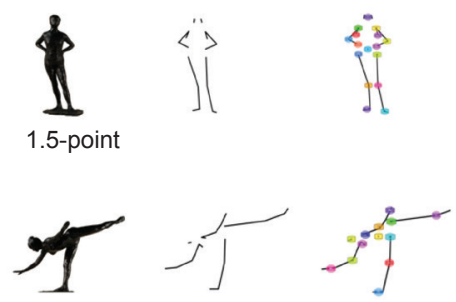

6.0-point
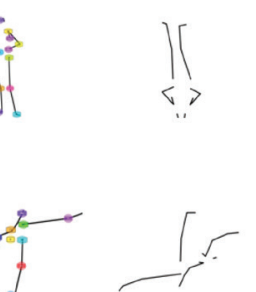

Inverted Position
Figure 4. The stimuli reduced to lines used in Experiment 2: 1.5-point (A) and 6.0-point (B) in the original position group and inverted position group. The same AOIs used in Experiment 1 were used in Experiment 2 in both the original and inverted positions.

The same AOIs of Experiment 1 were used in Experiment 2. The stimuli were randomized for the participants in the OP and IP groups. The training stimulus (4.0 points) was also reduced to lines and presented first to the participants to familiarize them with the experimental tasks.

\section{Results}

\section{Time estimations}

The ANOVA, considering the images (1.5- and 6.0-point stimuli reduced to lines) in the different groups (OP and IP), did not show an effect of image or group or an image $\times$ group interaction. The $t$-test results showed that the mean values of 37.12 and 37.42 s (OP group) and 34.24 and $34.03 \mathrm{~s}$ (IP group) for the 1.5- and 6.0-point stimuli were not different from the actual time of $36 \mathrm{~s}$ of image presentation.

\section{Eye movements}

Original position group. The ANOVA of FT, considering the images (1.5- and 6.0-point stimuli reduced to lines) and different image parts (areas), revealed a significant effect of image part $\left(F_{3,76}=86.55\right.$, $p<.001)$ and an image $\times$ image part interaction $\left(F_{3,76}=\right.$ $11.00, p<.001)$. The post hoc analyses showed that all of the parts of the images were distinctly observed (all $p<.01)$. The image $\times$ image part interaction showed that the areas that represented the head of the sculptures were more observed in the 1.5-point stimulus than in the 6.0-point stimulus, and the opposite was observed for the areas of images that represented the legs $(p<.001)$.

The ANOVA of the OC data showed a significant effect only of image part $\left(F_{3,73}=99.60, p<.001\right)$. Consistent with the results for the FT parameter, the post hoc comparisons revealed that all of the parts of the images were distinctly observed (all $p<.001$; Figure 5).

These results indicate that the participants tended to observe more the AOIs that represented the arms and legs than the head and torso in both the 1.5- and 6.0-point stimuli.
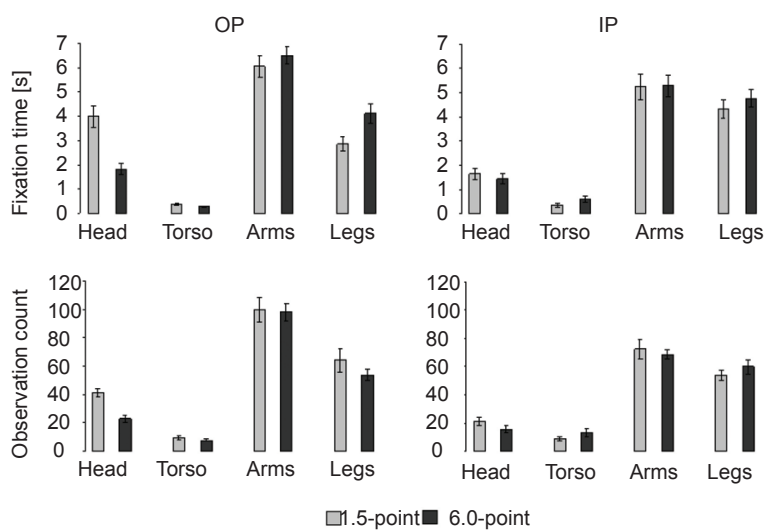

Figure 5. Mean fixation time and observation count for the head, torso, arms, and legs in the original position group (OP group) and inverted position group (IP group) for the 1.5- and 6.0-point stimuli reduced to lines.

Inverted position group. The ANOVA of the FT and OC data, considering the images (1.5- and 6.0-point stimuli reduced to lines) and different image parts (areas), only revealed a significant image $\times$ image part interaction $\left(F_{3,76}=68.03, p<.001\right.$, and $F_{3,76}=90.00$, $p<.001$, respectively). The post hoc analysis showed that all of the AOIs that represented the different parts of the sculptures were differently observed. The participants also tended to observe more the AOIs that represented the arms and legs than the head and torso in both stimuli (Fig. 5). This group tended to observe more the AOIs 
that represented the arms and legs than the head and torso in both stimuli.

The data analysis that compared the AOIs' order of observation time (TTF) did not reveal a significant effect in the OP or IP group. The participants spent the same time observing AOIs that represented the body parts in the 1.5- and 6.0-point stimuli (Figure $6)$.
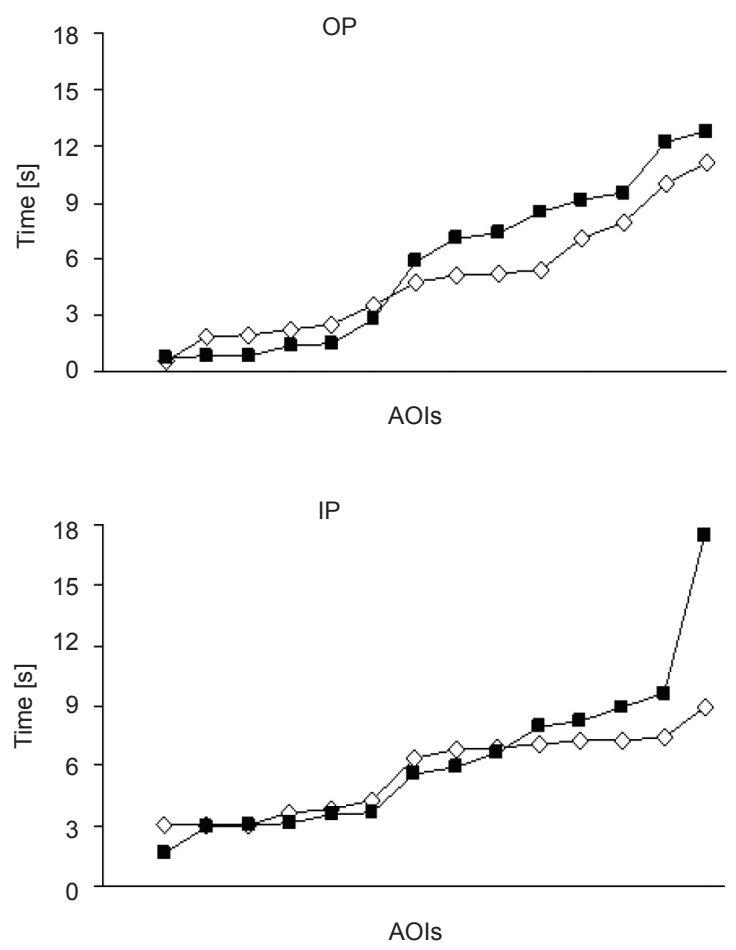

Figure 6. Time to first fixation for the 1.5- and 6.0-point stimuli reduced to lines in the original position group (OP) and inverted position group (IP).

\section{Discussion}

The ballerinas' body images reduced to lines did not affect time perception. In the distinct experimental conditions (OP and IP groups), no differences in time perception related to the actual time of exposure were found (36 s). However, eye movements were differentially affected by the images. Importantly, in the two experimental conditions, the images became abstract (Figure 4). Therefore, these abstract bodies affected movement perception. Abstract artwork that represent different abstract movements differentially activate specific brain areas related to visual perception (see Freedberg, \& Gallese, 2007; Kim, \& Black, 2007).

In Experiment 2, eye movements in both experimental conditions (OP and IP groups) were focused on the lines that represented the arms and legs of the dancers' bodies, regardless of the stimulus duration. Therefore, the eye movement analysis revealed that the participants' observation in Experiment 1 was related to the significance of the images (figurative content) more than the specific composition of the images (abstraction). Eye movements toward abstract paintings by Mondrian were also affected when the pictures were rotated by several degrees (Plumhoff, \& Schirillo, 2009). Different parts of one painting received more attention of the eyes according to the diagonal or horizontal lines. The same was observed in another study that investigated the effects of abstract image rotation (Vishwanath, \& Kowler, 2003). These results showed that perceptual and gaze localizations are based on the precise central reference position of images that coincides with their center of gravity.

From this perspective, the different abstract image content in the OP and IP groups generated different eye movements related to their specific compositional content. The eye movement results explain why the lines that represented the head and legs were distinctly observed by the participants, showing that the movement rather than the content of the image changes the pattern of ocular gaze.

\section{General discussion}

The present study showed that subjective time perception was affected by the compositional content of the images and their implied movement and by the duration of exposure (Experiment 1). The effects of different durations of exposure on time perception were also recently discussed by Nather, \& Bueno (2012b). Using 9-45 s exposure to the same ballerina images, the authors reported that time perception was related to implied movement when the images were presented, ranging from 27 to $36 \mathrm{~s}$. They indicated the necessity of conducting more parametric experimental studies that involve systematic comparisons of time perception across different task conditions and different durations because the perception of short and long durations involves different internal time and specific cognitive processes related to the properties of the stimuli used (Eagleman, 2008).

The specific focus of the present study was to understand the relationship between eye movement and time perception. The present results revealed aspects of both visual perception and time perception that affected aesthetic experience. Therefore, the use of images that contained different represented movements and evoked different perceptions caused by compositions (figurative and abstract images) must be considered in terms of the effects of visual movement perception on time estimation. The same images of Degas' ballerinas did not affect time perception when they were reduced to lines (Experiment 2). Although time distortions were not observed when the participants were exposed to the images for $18 \mathrm{~s}$, time distortions were evident when the participants were exposed to the images for $36 \mathrm{~s}$, replicating the data from previous studies (e.g., Nather, \& Bueno, 2011).

Eye movement patterns were similar in the G18 and G36 groups with regard to the FT and OC parameters, but they were different with regard to the TFF parameter. 
These results support the hypothesis that duration is a significant parameter in studies that involve the subjective time of artwork or aesthetic episodes (Cupchick, \& Gebotys, 1988; Nather, \& Bueno, 2012b). Interestingly, the eye movement data showed that differential attention was given to the images according to the significance of implicit body actions and body parts. Most of the participants used the strategy of carefully observing the image details to facilitate their mental repetition (imagery) during time estimations. Specifically, the individuals related in the post-study interviews that they repeated the same eye movements made while observing the ballerinas $(28 \%)$ or tried to "redraw" or "re-visualize" the body parts (50\%) in detail during the task of time estimation. This information (i.e., $78 \%$ of the individuals tried to remember the figurative body postures) was consistent with previous studies that showed that implied movement in two-dimensional images has more temporal markers of motion (Nather, \& Bueno, 2006), generating more sources of memory and producing a lengthening of the time experienced by memory storage (Ornstein, 1989).

Cutting (2002) described different aspects and constraints related to the perception of movement in static artwork (paintings and sculptures). He discussed that artists use at least five different criteria to represent motion, such as blurring the background of the scene, superimposing different instants of a moving object in the same painting, and using asymmetries generated by relationships between human body parts in different ways (head, torso, arms, and legs). From the perspective of visual motion perception, the 1.5- and 3.0-point stimuli were more symmetrical than the 6.0-point stimulus. Therefore, the eye movements could be an appropriate indicator of motion perception in static images because the participants were more attentive to the legs and arms in the stimuli that implied more movement (6.0 points). Therefore, the participants tended to increase the observation of legs according to the movement intensity of this stimulus when they were exposed to it for $36 \mathrm{~s}$. Artwork pieces that represent figurative forms, such as body postures, can be discussed in terms of their most evident indicators. Body postures reveal implicit realism evoked by themselves (e.g., balance in three-dimensional space). Therefore, the relationships between the legs' positions and body as a whole could indicate an actual displacement in two-dimensional movement representations by memory of the real threedimensional world because of the center of gravity.

The realism of these Degas' ballerinas that represent motion is evident in the artwork literature (e.g., Growe, 2001). The present study showed that eye movements were more dynamic (i.e., quick) when the participants observed the 6.0-point stimulus (more movement) for $36 \mathrm{~s}$. Under this experimental condition, eye fixation tended to be more specifically oriented to specific body parts, depending on their implied movement suggested as a whole. This suggests that the implicit movement of the ballerinas was more repeatedly visualized, thus enabling the subjects to imagine or better remember the body shapes (Brandt, \& Stark, 1997; Laeng, \& Teodorescu, 2002). Brain areas are more activated and repeatedly recruited when individuals attempt to produce a better schematic signal of the observed action (Daems, \& Verfaille, 1999; Reed, \& Farah, 1995; Rizzolatti, \& Craighero, 2004), which was evident in the present study when the participants were exposed to the stimuli for $36 \mathrm{~s}$.

From this perspective, eye movement repetition could be interpreted as mental reconstruction of a ballerina body's index, which was suggested by Nather et al. (2011) who used short durations (milliseconds) with a bisection paradigm of time estimation, in which the participants were exposed to the images several times. Thus, attention to body parts by repetition produced more stored pulses in memory (Gibbon et al., 1984). These repetitions of observation may occur on a continuum of time to successively reset the internal clock. Therefore, the data in the present study allow us to infer that the internal clock runs faster and turns off repeatedly during 18 or 36 s exposures. Therefore, during the time estimations, the participants compared in memory these stored pulses to extrapolate the actual time experienced and unconsciously counted time without making actual numeric counts. This hypothesis was supported by the $18 \mathrm{~s}$ exposure data, in which time distortions were not observed, likely because of the turning off of the internal clock was the same for the different ballerina movements. However, the images reduced to lines did not change time perception because these compositions were not specifically related to body movements. Therefore, the results of Experiment 2 (OP and IP groups) must be considered in terms of their abstract content rather than movement perception because the patterns of eye movements were affected by the simple rotation of the artwork or image (Plumhoff, $\&$ Schirillo, 2009). These kinds of abstract compositions did not turn off the internal clock because they did not represent the motion of human beings.

Embodiment mechanisms in artwork associated with time perception must be considered. Nather et al. (2011) showed that the duration was judged to be shorter for a 1.5-point stimulus than for a 6.0-point stimulus when the ballerinas' bodies were observed several times, from 0.4 to $1.6 \mathrm{~s}$ but not from 2 to $8 \mathrm{~s}$, in a bisection paradigm. Importantly, in this previous study, the participants observed the images repeatedly (i.e., approximately 60 times each) to decide their duration several times by comparing them to the median duration. According to Nather et al., based on embodiment mechanisms and arousal effects, time distortions in the perceiver may be an index of empathic processes that enable the individual to understand the actions of others as a function of timing related to the observed movement. The data in the present study support this idea because the participants reported in the post-study interviews that they repeated the ballerinas' images in their mind. However, in the present study, the images 
were shown only once (reproduction paradigm; Block, 1990). This procedure of time estimation suggests that the ballerinas' bodies were repeated several times in the mind when the subjects observed them and also when they were estimating time. By analogy, this mental image repetition may also be an encephalic index of brain activation.

The brain areas recruited by the observation of other body postures that imply different human movements were described in studies that used pictures of people and artwork that represented human figures (Kourtzi, \& Kanwisher, 2000; Osaka, Matsuyoshi, Ikeda, \& Osaka, 2010). Other recent works have used time as a parameter to understand the processes associated with the visual perception of movement in static images (Orgs et al., 2011). These works showed that implied motion recruited the same brains areas involved in real movement perception in both motion processing and the integration of motion and spatial position, which depends on setting the processing of body forms (i.e., the image exposure context). Moscatelli et al. (2011) found that pictures of people making dynamic movements were more precisely estimated, but the authors did not discuss the importance of the background of the scenes' pictures on time perception. Body postures isolated on a background have shown that scenes that imply the movement of human beings affect subjective time perception (Nather, \& Bueno, 2012a). Therefore, the effects of the contextual composition image exposure and experimental design must be considered in studies of time perception (Experiments 1 and 2).

The present study used long-term durations (18 or $36 \mathrm{~s}$ ), and the participants were exposed to the stimuli once. The results showed that time perception was distorted in different ways, consistent with previous studies that used the same images and same paradigm of time estimation (reproduction). For the $18 \mathrm{~s}$ exposure duration, the unconscious mental repetition of images was likely not sufficient to distort time, which was observed for the $36 \mathrm{~s}$ exposure duration. The real repetition of these images using a short-term duration (milliseconds) in the bisection paradigm showed that the subjects repeated the images several times because of the procedure (Nather el al., 2011). The present study used 18 and $36 \mathrm{~s}$ exposure, and the implied movement of the static images was repeated in the participants' minds several times but not really re-visualized. Therefore, this important information explains why the unmoving ballerinas (less movement) caused less distortion than the moving ballerinas (more movement). To explain these results, we propose a hypothesis that is in agreement with the clock model (Gibbon et al., 1984). The pulses of the internal clock run faster for images that imply more movement. However, the clock could be turned off several times because of the duration of exposure to the stimuli (18 and $36 \mathrm{~s}$ ). Therefore, during the observation of images and consequently during the experiment, the individuals estimated time according to the dynamics of eye movements evoked by body positions. This hypothesis may be supported by studies that show that movement perception in two-dimensional static images modulates both eye movement and subjective time.

\section{Conclusion}

The present study showed that eye movements were modulated by motion perception in static images (figurative artwork). Eye movements can be used as a dynamic index that explains how movement is processed by people to generate time distortions. Time distortions in the perceiver may be hypothesized to be an index of empathy by arousal-level processes related to the repetition of body postures by the eyes. This index enables individuals to better understand the actions of others through embodiment mechanisms. First, a body position that represents more movement induces rapid eye movements. Second, by neural activation of eye movement repetition, individuals reconstruct the observed movements during the tasks of image observation and time estimation. Third, this movement repetition is implicitly related to the imagery of the continuation of the observed movements of the differential body postures that generate differential storage of the internal clock pulses.

\section{Acknowledgements}

This research was supported by a Post-Doctoral Scholarship from CAPES to F.C. Nather and the CAPES-COFECUB Program (Brazil-France) to J.L.O. Bueno and E. Bigand. The work was also funded by grants from CNPq to J.L.O. Bueno and from the French National Research Agency. F.C. Nather thanks Chloé Fourier for help with the Tobii Studio methodological procedure.

\section{References}

Angrilli, A., Cherubini, P., Pavese, A., \& Manfredini, S. (1997). The influence of affective factors on time perception. Perception and Psychophysics, 59, 972-982.

Arnheim, R. (2004). Art and visual perception: a psychology of the creative eye. Berkeley:University of California Press.

Block, R. A. (1990) Models of psychological time. In: R. A. Block (Ed.), Cognitive models of psychological time (pp. 1-35). Hillsdale, N.J.: Lea Publishers.

Brandt, S. A., \& Stark, L. W. (1997). Spontaneous eye movements during visual imagery reflect the content of the visual scene.Journal of Cognitive Neuroscience, 9, 27-38.

Cupchick, G. C. (1976). Perspective théorique et empirique sur peintures impressioniste. Bulletin de Psychologie, 36, 720-729.

Cupchick, G. C., \& Gebotys, R. (1988). The experience of time, pleasure, and interest during aesthetic episodes. Empirical Studies of the Arts, 6, 1-12.

Cutting, J. E. (2002). Representing motion in a static image: constraints and parallels in art, science, and popular culture. Perception, 31, 1165-1193.

Daems, A., \& Verfaillie, K. (1999). Viewpoint-dependent priming effects in the perception of human actions and body postures. Visual Cognition, 6, 665-693.

De'Sperati, C., \& Santandrea, E. (2005). Smooth pursuit-like eye movements during mental extrapolation of motion: the facilitatory effect of drowsiness. Cognitive Brain Research, 25, 328-338. 
Droit-Volet, S., \& Gil, S. (2009). The time-emotion paradox. Journal of Philosophical Transactions of the Royal SocietyB. Biological Sciences, 364, 1943-1953.

Duchowski, A. T. (2007). Eye tracking methodology: theory and practice. London: Springer.

Eagleman, D. M. (2008). Human time perception and its illusions. Current Opinion in Neurobiology, 18, 131-136.

Flanagan, J. R., \& Johansson, R. S. (2003). Action plans used in action observation. Nature, 14(424), 769-771.

Freedberg, D., \& Gallese, V. (2007). Motion, emotion and empathy in esthetic experience. Trends in Cognitive Sciences, 11, 197-203.

Gallese, V. (2005). Embodied simulation: from neurons to phenomenal experience. Phenomenology Cognitive Sciences, 4, 23-48.

Gibbon, J., Church, R. M., \& Meck, W. H. (1984). Scalar timing in memory. In: J. A. Gibbon, \& L. Allan (Eds.), Timing and time perception (series title: Annals of the New York Academy of Sciences, vol. 423; pp. 52-77). New York: New York Academy of Sciences.

Growe, B. (2001). Edgar Degas, 1834-1917. Koln: Taschen.

Gueugneau, N., Crognier, L., \& Papaxanthis, C. (2008). The influence of eye movements on the temporal features of executed and imagined arm movements. Brain Research, 1187, 95-102.

Henderson, J. M. (1993) Eye movement control during visual object processing: effects of initial fixation position and semantic constraint. Canadian Journal of Experimental Psychology, 47, 7998.

Henderson, J. M. (2003). Human gaze control during real-world scene perception. Trends in Cognitive Sciences, 7, 498-504.

Kapoula, Z., \& Gaertner, C. (2013). Motion and lateral organization in Monet's paintings impact body sway. Perception, in press.

Kim, C. Y., \& Blake, R. (2007). Brain activity accompanying perception of implied motion in abstract paintings.Spatial Vision, 20, 545-560.

Kourtzi, Z., \& Kanwisher, N. (2000). Activation in human MT MST by static images with implied motion. Journal of Cognitive Neuroscience, 12, 48-55.

Laeng, B., \& Teodorescu, D. S. (2002). Eye scanpaths during visual imagery reenact those of perception of the same visual scene. Cognitive Science, 26, 207-231.

Mannan, S. K., Ruddock, K.H., \&Wooding, D. S. (1997). Fixation patterns made during brief examination of two-dimensional images. Perception, 26, 1059-1072.

Moscatelli, A., Polito, L., \&Lacquaniti, F. (2011). Time perception of action photographs is more precise than that of still photographs. Experimental Brain Research, 210, 25-32.

Nather, F. C. (2006). Percepção de movimento e tempo subjetivo nas artes visuais. Unpublished doctoral dissertation. São Paulo: University of São Paulo.

Nather, F. C., \& Bueno, J.L.O. (2006). Efeito de imagens estáticas com diferentes representações de movimento sobre a percepção subjetiva de tempo. Psicologia: Reflexão, \& Crítica, 19, 217-224.

Nather, F. C., \& Bueno, J. L. O. (2008). Movement ranking scale of human body static images for subjective timing estimation. Proceedings of the Annual Meeting of the Society for Psychophysics, 24, 185-190.

Nather, F. C., \& Bueno, J. L. O. (2011). Static images with different induced intensities of human body movements affect subjective time. Perceptual and Motor Skills, 113, 155-170.

Nather, F. C., \& Bueno, J. L. O. (2012a) Timing perception in paintings and sculptures of Edgar Degas. Kronoscope: The Journal for the Study of Time, 12, 16-30.

Nather, F. C., \& Bueno, J. L. O. (2012b). The presentation of long term duration of body movement in impressionist artworks differently distorts the perception of time. Proceedings of the Annual Meeting of the International Society for Psychophysics, 28, 286-291.

Nather, F. C., Bueno, J. L. O., Bigand, E., \& Droit-Volet, S. (2011). Time changes with the embodiment of another's body posture. PLoS One, 6, e19818.

Nodine, C. F., Locher, P. J., \& Krupinski, E. A. (1993). The role of formal art training on perception and aesthetic judgment of art compositions, Leonardo, 26, 219-227.

Orgs, G., Bestmann, S., Schuur F., \& Haggard, P. (2011). From body form to biological motion: the apparent velocity of human movement biases subjective time. Psychological Science, 22, 712 717.

Orgs, G., Kirsh, L., \& Haggard, P. (2013). Time perception during apparent biological motion reflects subjective speed of movement, not objective rate of visual stimulation. Experimental Brain Research, 227, 223-229.

Ornstein, R. E. (1969). On the experience of time. Harmondsworth: Penguin.

Osaka, N., Matsuyoshi, D., Ikeda, T., \& Osaka, M. (2010). Implied motion because of instability in Hokusai Manga activates the human motion-sensitive extrastriate visual cortex: an fMRI study of the impact of visual art. Neuroreport, 21, 264-267.

Pavan, A., Cuturi,L. F., Maniglia, M., Casco, C., \& Campana, G. (2011). Implied motion from static photographs influences the perceived position of stationary objects. Vision Research, 51, 187194.

Plumhoff, J. E., \& Schirillo, J. A. (2009). Mondrian, eye movements, and the oblique effect. Perception, 38, 719-731.

Rayner, K. (1998). Eye movements in reading and information processing: 20 years of research. Psychological Bulletin, 124, 372422.

Reed, C. L., \& Farah, M. J. (1995). The psychological reality of the body schema: a test with normal participants. Journal of Experimental Psychology: Human Perception and Performance, 21, 334-343.

Rizzolatti, G., \& Craighero, L. (2004). The mirror-neuron system. Annual Review of Neuroscience, 27, 169-192.

Robinette, K. M., Balckwell, S., Daanen, H., Boehmer, M., Fleming, S., Brill, T., Hoeferlin, D., \& Beurnsides, D. (2002). Civilian American and European Surface Anthropometry Resource (CAESAR), Final Report: Volume I. Summary. United States Air Force Research Laboratory, Human Effectiveness Directorate.

Tse, P. U., Intriligator, J., Rivest, J., \& Cavanagh, P. (2004). Attention and the subjective expansion of time. Perception and Psychophysics, 66, 1171-1189.

Vishwanath, D., \& Kowler, E. (2003). Localization of shapes: eye movements and perception compared. Vision Research, 43, 1637 1653.

Vogt, S., \& Magnussen, S. (2007). Expertise in pictorial perception: eye-movement patters and visual memory in artists and laymen. Perception, 36, 91-100.

Wang, H. X., Freeman, J., Merriam, E. P., Hasson, U., \& Heeger, D. J. (2012). Temporal eye movement strategies during naturalistic viewing. Journal of Vision, 12, 16.

Wooding, D. S. (2002). Fixation maps: quantifying eye-movement traces. Proceedings of the Eye Tracking Research, \& Applications Symposium, 2002, 31-36.

Yamamoto, K., \& Miura, K. (2012). Time dilation caused by static images with implied motion. Experimental Brain Research, 223, 311-319.

Zangemeister, W. H., Sherman, K., \& Stark, L. (1995). Evidence for a global scanpath strategy in viewing abstract compared with realistic images. Neuropsychologia, 33, 1009-1025. 